\title{
An affibody in complex with a target protein: Structure and coupled folding
}

\author{
Elisabet Wahlberg ${ }^{\dagger}$, Christofer Lendel ${ }^{\dagger}$, Magnus Helgstrand, Peter Allard, Vildan Dincbas-Renqvist, Anders Hedqvist, \\ Helena Berglund, Per-Åke Nygren, and Torleif Härd ${ }^{\ddagger}$
}

Department of Biotechnology, Royal Institute of Technology, S-106 91 Stockholm, Sweden

Edited by Adriaan Bax, National Institutes of Health, Bethesda, MD, and approved December 27, 2002 (received for review October 9, 2002)

\begin{abstract}
Combinatorial protein engineering provides powerful means for functional selection of novel binding proteins. One class of engineered binding proteins, denoted affibodies, is based on the three-helix scaffold of the $Z$ domain derived from staphylococcal protein $A$. The $Z_{\text {SPA-1 }}$ affibody has been selected from a phagedisplayed library as a binder to protein A. Z micromolar affinity to its own ancestor, the $Z$ domain. We have characterized the $Z_{\text {SPA-1 }}$ affibody in its uncomplexed state and determined the solution structure of a $Z: Z_{S P A-1}$ protein-protein complex. Uncomplexed $Z_{\text {SPA-1 }}$ behaves as an aggregation-prone molten globule, but folding occurs on binding, and the original ( $\mathrm{Z}$ ) three-helix bundle scaffold is fully formed in the complex. The structural basis for selection and strong binding is a large interaction interface with tight steric and polar/nonpolar complementarity that directly involves 10 of 13 mutated amino acid residues on $Z_{\text {SPA-1. }}$. We also note similarities in how the surface of the $Z$ domain responds by induced fit to binding of $Z_{S P A-1}$ and Ig FC, respectively, suggesting that the $Z_{\text {SPA-1 }}$ affibody is capable of mimicking the morphology of the natural binding partner for the $Z$ domain.
\end{abstract}

protein engineering | protein-protein interactions | molecular recognition | NMR spectroscopy | induced fit

$\mathbf{T}^{\mathrm{T}}$ here is an interest in generating novel classes of binding proteins that can be used as an alternative to immunoglobulins in various biochemical assays and biotechnological applications. To this end, carefully chosen protein domains can be used as framework structures for combinatorial protein engineering. Affibodies constitute a class of engineered binding proteins for which the three-helix bundle $\mathrm{Z}$ domain is used as a scaffold. The 58-aa residue $\mathrm{Z}$ domain is derived from one of five homologous domains (the B domain) in Staphylococcus aureus protein A (SPA). SPA binds strongly to the Fc region of immunoglobulins, and $\mathrm{Z}$ was originally developed as a stabilized gene fusion partner for affinity purification of recombinant proteins by using IgG-containing resins (1). The structure of a complex between the B domain of SPA and an Fc fragment shows that the binding surface consists of residues that are exposed on helices 1 and 2, whereas helix 3 is not directly involved in binding (2). Affibodies are selected from combinatorial libraries in which typically 13 residues at the Fc-binding surface of helices 1 and 2 are randomized. Specific binders to target proteins are then identified by biopanning the phage-displayed library against desired targets (3). Several Z-based affibodies with specific proteinbinding properties have in this way been developed and used as affinity tools in a number of applications (4-7).

Structural studies of engineered protein-binding domains and their complexes are of interest for methods development in biotechnology as well as for basic studies of protein-protein interactions and the mechanisms of biomolecular recognition. Here we describe the (solution) structural and biophysical properties the $\mathrm{Z}_{\mathrm{SPA}-1}$ affibody (Fig. 1), which was isolated using its ancestor protein SPA as panning target during selection (8). We have also determined the structure of a complex between $\mathrm{Z}_{\mathrm{SPA}-1}$ and the wild-type $\mathrm{Z}$ domain, which for these studies is representative of SPA. The experiments reveal an intricate mechanism for molecular recognition that involves both coupled folding of the $Z_{\text {SPA-1 }}$ affibody and conformational adaptation (induced fit) of side chains at the surface of the $\mathrm{Z}$ domain. The structure of the complex clearly shows why and how this affibody was selected from the library, and our results provide inspiration for improvements in the design of combinatorial libraries and selection of strong binders.

\section{Methods}

NMR Spectroscopy. Protein preparation and NMR resonance assignments are described elsewhere (9). NMR samples for structure determination contained $1.5-2.0 \mathrm{mM}{ }^{13} \mathrm{C},{ }^{15} \mathrm{~N}$-labeled $\mathrm{Z}$ (or $\mathrm{Z}_{\text {SPA-1 }}$ ), and $10-25 \%$ excess of unlabeled $\mathrm{Z}_{\text {SPA-1 }}$ (or $\mathrm{Z}$ ) in $20 \mathrm{mM}$ potassium phosphate $/ 0.01 \% \mathrm{NaN}_{3} / 10$ or $100 \% \mathrm{D}_{2} \mathrm{O}$ at $\mathrm{pH} 5.6$ (uncorrected reading). NMR data were acquired at $30^{\circ} \mathrm{C}$ on Bruker (Billerica, MA) Avance 500 and $600 \mathrm{MHz}$ and Varian Inova $800 \mathrm{MHz}$ spectrometers equipped with $5-\mathrm{mm}$ triple resonance probes. Stereospecific assignments of $\mathrm{H}^{\beta}$ protons and $\chi_{1}$ dihedral angles (10) were obtained for most nonoverlapping $\mathrm{H}^{\beta}$ pairs. Isotope-edited and -filtered NOESY experiments $(11,12)$ were recorded with a cross-relaxation mixing time of $100 \mathrm{~ms}$ (at $800 \mathrm{MHz}$ ) or $130 \mathrm{~ms}(500$ and $600 \mathrm{MHz})$. Hydrogen bond donors were identified by slow amide proton exchange. Nuclear Overhauser effect (NOE) assignments were obtained using ANSIG for WINDOWS (13).

Structure Determination. Distance restraints were derived from NOE cross-peak volumes, which were adjusted for multiplicity and classified as interproton distances $<2.7,<3.5$, or $<5 \AA$ based on known distances in $\alpha$-helices (14). Restraints involving groups of equivalent or nonstereospecifically assigned diastereotopic protons were adjusted or discarded (15). Backbone dihedral angle restraints were derived from chemical shifts by using the program TALOS (16). Some $\varphi$ angles that could not be assigned values based on chemical shifts were restrained to negative values based on very weak intraresidue $\mathrm{d}_{\mathrm{HNH} \alpha} \mathrm{NOE}$ connectivities (17). Hydrogen bonds were included based on the exchange experiments, with acceptor oxygen to donor hydrogen and nitrogen distances set to $1.8-2.4$ and 2.6-3.2 $\AA$, respectively (18).

Structures were calculated with XPLOR-NIH (http:// nmr.cit.nih.gov/xplor-nih/) by using ab initio simulated annealing with $r^{-6}$ averaging (19) of distances involving nonstereospecifically assigned protons. A pseudopotential for the radius of gyration (20) was used on residues 3-56 in both proteins to improve packing, and a conformational database potential (21) was used to improve dihedral angle distributions. The refinement was performed with a full Lennard-Jones potential and an

This paper was submitted directly (Track II) to the PNAS office.

Abbreviations: SPA, Staphylococcus aureus protein A; ANS, 8-anilino-1-naphthalenesulfonic acid; NOE, nuclear Overhauser effect.

Data deposition: The atomic coordinates have been deposited in the Protein Data Bank www.rcsb.org (1h0t).

${ }^{\dagger} E . W$. and C.L. contributed equally to this work.

¥To whom correspondence should be addressed. E-mail: torleif.hard@biotech.kth.se. 

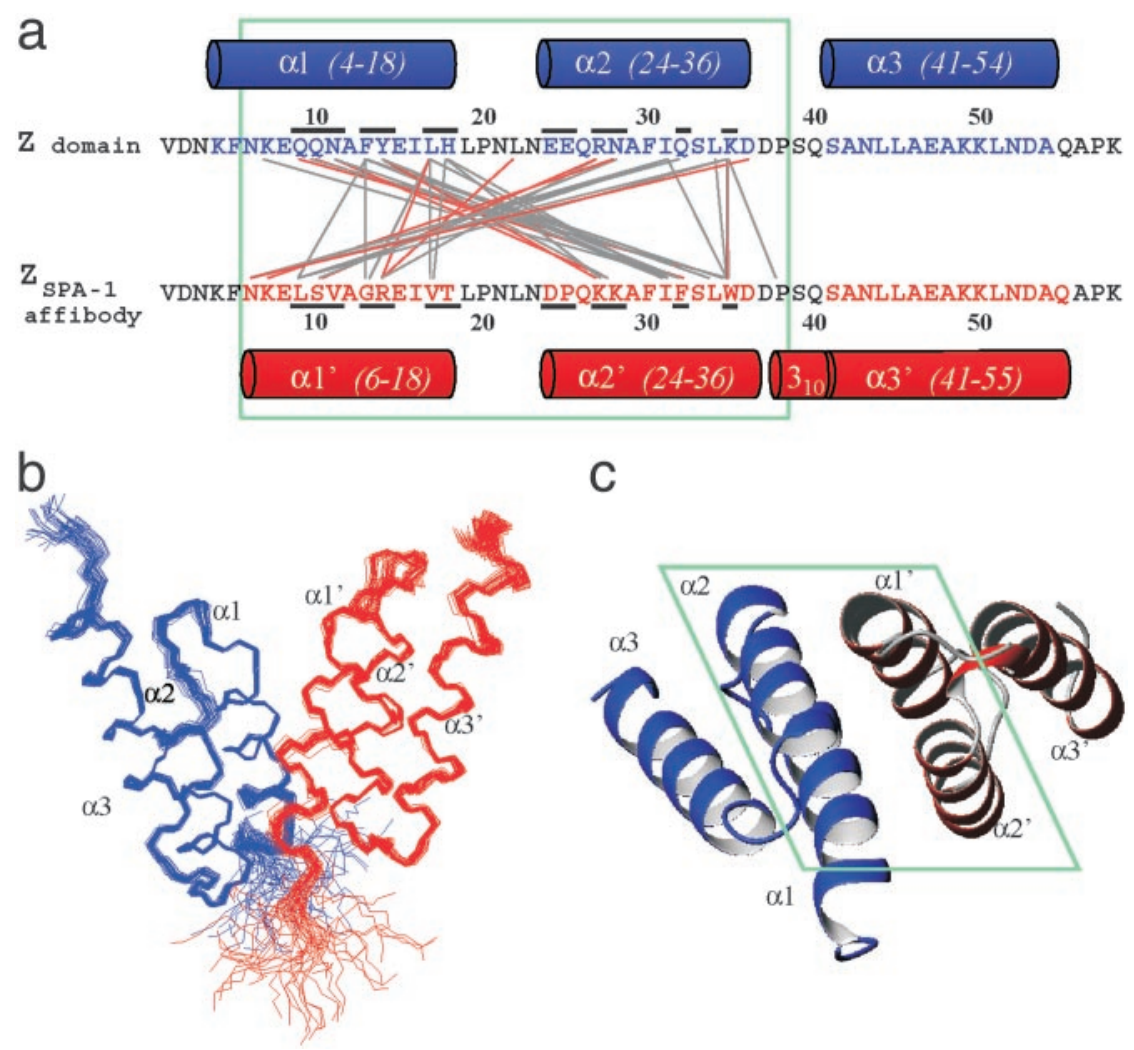

C

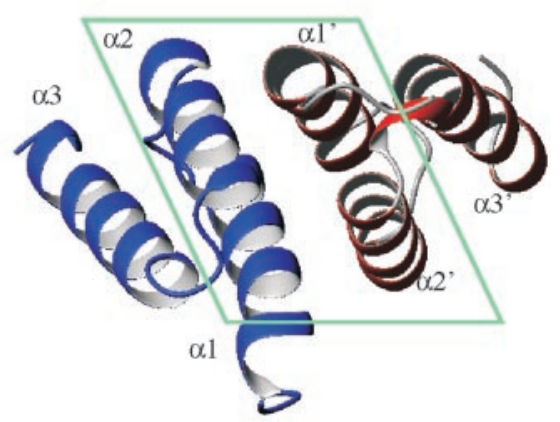

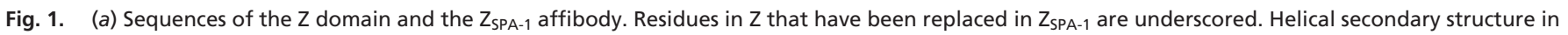

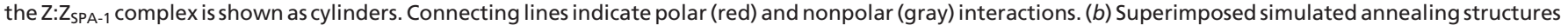

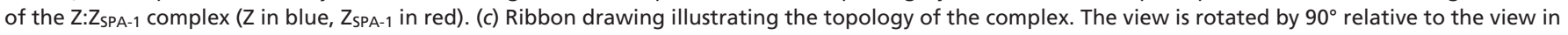

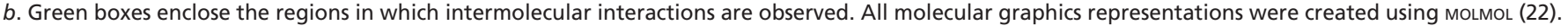

electrostatic interaction potential that was reduced to $10 \%$ to compensate for the fact that the refinement was carried out in a vacuum. An ensemble of 100 structures was calculated, and 40 of these were chosen based on restraint violation and Ramachandran statistics and analyzed using MOLMOL (22), PROCHECK-NMR (23), and WHAT IF (24) (Table 1).

Optical Spectroscopy. CD was measured on a Jasco (Easton, MD) J-810 spectropolarimeter. ANS (8-anilino-1-naphthalenesulfonic acid, Sigma) fluorescence was measured at room temperature on a Perkin-Elmer Luminescence Spectrometer, Model LS50B.

\section{Results and Discussion}

The Uncomplexed $\mathbf{Z}_{\text {SPA-1 }}$ Affibody Is a Molten Globule. The biophysical properties of $Z_{\text {SPA-1 }}$ are illustrated in Fig. 2. The ${ }^{1} \mathrm{H}-{ }^{15} \mathrm{~N}$ heteronuclear single quantum correlation NMR spectrum (Fig. $2 a$ ) shows poor dispersion in the amide region and substantial broadening of several resonances. These features are characteristic of a protein in which interconversion between multiple poorly packed conformations on different time scales leads to averaging and differential line broadening. The NMR spectrum of $\mathrm{Z}_{\mathrm{SPA}-1}$ at low $(50 \mu \mathrm{M})$ concentrations or at different $\mathrm{pH}$ values has the same appearance. However, addition of the $\mathrm{Z}$ domain to $\mathrm{Z}_{\mathrm{SPA}-1}$ samples results in a drastic improvement in the quality of the NMR spectrum, with good dispersion and sharp resonances (Fig. 2a). This suggests that $Z_{\text {SPA-1 }}$ adopts a well defined conformation in complex with the $\mathrm{Z}$ domain.

The CD spectrum of $\mathrm{Z}_{\mathrm{SPA}-1}$ (Fig. $2 b$ ) is consistent with a predominantly $\alpha$-helical conformation at low $(25-50 \mu \mathrm{M})$ concentrations. However, a comparison with the CD spectrum of the

\section{Table 1. Restraints and structure statistics}

\begin{tabular}{|c|c|}
\hline \multicolumn{2}{|l|}{ Nonredundant NOEs } \\
\hline Z domain & 1,008 \\
\hline$Z_{\text {SPA-1 }}$ affibody & 945 \\
\hline Intermolecular & 238 \\
\hline Dihedral angles, $\phi / \psi / \chi$ & $73 / 73 / 30$ \\
\hline Hydrogen bonds in $\alpha$-helices & 82 (for $41 \mathrm{H}$ bonds) \\
\hline Total number of restraints & 2,449 (21.1 per residue) \\
\hline XPLOR van der Waals energy & $-1,495 \pm 32$ \\
\hline \multicolumn{2}{|l|}{ rms deviation from restraints* } \\
\hline Distances, $\AA$ & $0.063 \pm 0.001$ \\
\hline Torsion angles, $^{\circ}$ & $0.16 \pm 0.07$ \\
\hline \multicolumn{2}{|l|}{ Deviation from ideal stereochemistry* } \\
\hline Bonds, $\AA$ & $0.0091 \pm 0.0001$ \\
\hline Angles, ${ }^{\circ}$ & $1.01 \pm 0.03$ \\
\hline Impropers, $^{\circ}$ & $0.85 \pm 0.02$ \\
\hline \multicolumn{2}{|l|}{ Ramachandran statistics, \%* } \\
\hline Most favorable regions & 93.2 \\
\hline Allowed regions & 6.8 \\
\hline \multicolumn{2}{|l|}{ Coordinate precision, $\AA^{* \dagger}$} \\
\hline Backbone & $0.34 \pm 0.07$ \\
\hline Heavy atoms & $0.70 \pm 0.07$ \\
\hline \multicolumn{2}{|l|}{ Structure $\mathrm{Z}$ scores $^{\ddagger}$} \\
\hline Second generation packing quality & 0.25 \\
\hline Ramachandran plot appearance & -0.47 \\
\hline$\chi_{1} / \chi_{2}$ rotamer normality & -2.22 \\
\hline Bond lengths/angles & $1.336 / 1.082$ \\
\hline
\end{tabular}

*For ensemble of 40 structures.

${ }^{\dagger}$ Residues 8-56 in Z and ZSPA-1.

‡Using WHAT IF (24); representative structure ( $\mathrm{nr} 1 \mathrm{in} 1 \mathrm{~h} 0 \mathrm{t}$ ). 

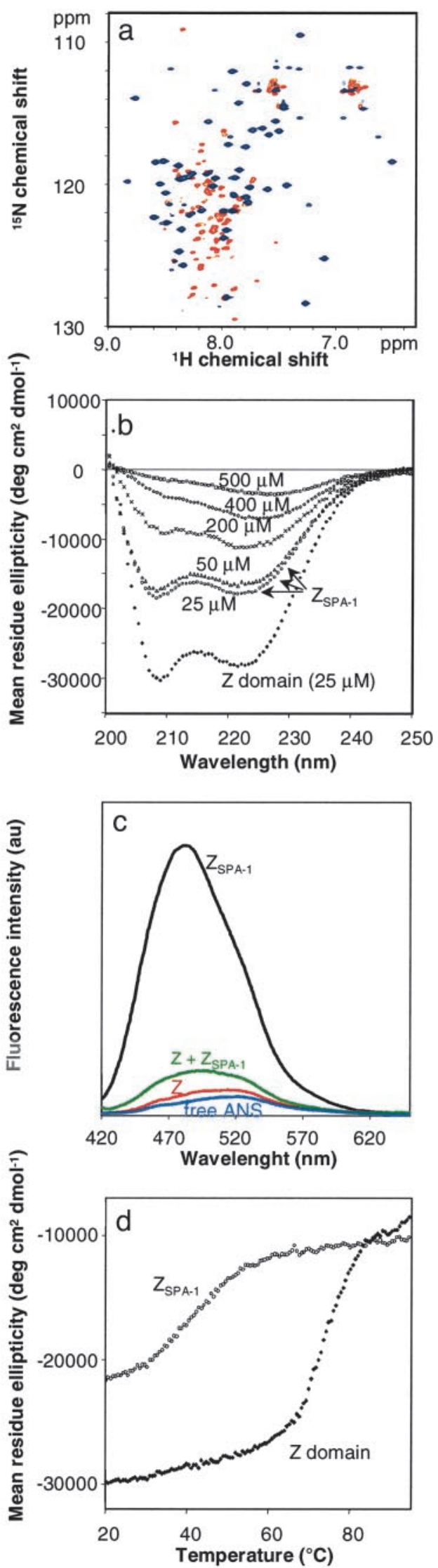

Fig. 2. Biophysical characterization of the $Z_{\text {SPA-1 }}$ affibody. (a) Section of the ${ }^{1} \mathrm{H}-{ }^{15} \mathrm{~N}$ heteronuclear single quantum correlation spectrum of ${ }^{15} \mathrm{~N}$-labeled $Z_{\text {SPA-1 }}$ in the absence (red) and presence (blue) of the unlabeled $Z$ domain.
$\mathrm{Z}$ domain shows that the mean residue ellipticity is only about $60 \%$ of the value expected for a fully folded three-helix bundle. The poor quality of the NMR spectrum prevents further analysis of the secondary structure of uncomplexed $\mathrm{Z}_{\text {SPA-1 }}$, and it is not possible to say, for instance, whether only two of three helices are formed or whether the CD spectrum reflects some other kind of averaging of the helix content.

To test the possibility that $\mathrm{Z}_{\mathrm{SPA}-1}$ is a molten globule, we examined the binding properties of ANS (Fig. $2 c$ ). There is a large enhancement of ANS fluorescence with $\mathrm{Z}_{\text {SPA-1 }}$, whereas the dye appears not to bind to the $\mathrm{Z}$ domain and only weakly to a $Z_{\text {SPA-1 }}: Z$ mixture. ANS binding and consequent fluorescence enhancement are characteristic properties of a molten globule (25), which contains some secondary structure but in which the hydrophobic core is only loosely packed $(26,27)$. Decreased stability and loss of packing in $\mathrm{Z}_{\mathrm{SPA}-1}$ is also apparent in thermal melting profiles, which show a significantly lower melting temperature for $\mathrm{Z}_{\mathrm{SPA}-1}\left(T_{\mathrm{M}} \approx 40^{\circ} \mathrm{C}\right)$ than for the $\mathrm{Z}$ domain $\left(T_{\mathrm{M}} \approx\right.$ $75^{\circ} \mathrm{C}$ ) and a less cooperative melting transition in the case of $\mathrm{Z}_{\mathrm{SPA}-1}$ (Fig. $2 d$ ). At concentrations approaching the millimolar range, $\mathrm{Z}_{\mathrm{SPA}-1}$ also undergoes self association, as evidenced by a concentration dependence of the CD spectrum (Fig. $3 b$ ) and an apparent molecular weight corresponding to a dimer in gel filtration elution profiles of concentrated samples (not shown). The change in the CD spectrum indicates further loss of helix content on self-association.

The $\mathbf{Z}_{\text {SPA-1 }}$ Affibody Folds on Binding to the $\mathbf{Z}$ Domain. $Z_{\mathrm{SPA}-1}$ binds to $Z$ with a dissociation constant $K_{\mathrm{d}}=6 \mu \mathrm{M}$ (8). Recognition and binding are coupled to protein folding; $Z_{\mathrm{SPA}-1}$ clearly adopts the three-helix bundle topology of the wild-type $\mathrm{Z}$ domain in the $\mathrm{Z}_{\text {SPA-1 }}: \mathrm{Z}$ complex (Fig. 1). The only differences in secondary structure between the two proteins in the complex are that helix 1 of $Z_{\text {SPA-1 }}$ appears to be slightly shorter at the $\mathrm{N}$-terminal due to fraying, and that residues 38-40 between helices 2 and 3 more clearly adopt a 310 helical conformation. The backbone rms difference of residues $8-56$ in the two proteins is $0.9 \AA$; side chain conformations and packing within the cores are very similar. The structural properties of wild-type $\mathrm{Z}$ are therefore preserved in the $\mathrm{Z}_{\mathrm{SPA}-1}$ affibody when it adopts its folded conformation in the complex.

The lower stability of $\mathrm{Z}_{\mathrm{SPA}-1}$ can be explained by polar-tononpolar side chain mutations at the surface, because a large fraction of the surface that would be exposed in the folded $Z_{\text {SPA-1 }}$ has been made nonpolar. For instance, Gln-9, Asn-11, Glu-25, Gln-32, and Lys-35 in Z have been replaced by *Leu-9, *Val-11, ${ }^{*}$ Pro-25, *Phe-32, and ${ }^{*}$ Trp-35 in $\mathrm{Z}_{\mathrm{SPA}-1}$, whereas replacement of nonpolar side chains by polar ones has occurred only for Phe-13 and Tyr-14 in Z, which are replaced by *Gly-13 and *Arg-14 in $\mathrm{Z}_{\mathrm{SPA}-1}$. (We denote residues that are replaced in $\mathrm{Z}_{\mathrm{SPA}-1}$ with ${ }^{*}$.) An estimate of the ratio of polar-to-nonpolar surfaces in the two molecules based on the structure of the complex yields $45: 55 \%$ and $36: 64 \%$ for $\mathrm{Z}$ and $\mathrm{Z}_{\mathrm{SPA}-1}$, respectively. The surface properties of uncomplexed affibodies might therefore be considered in the optimization of combinatorial libraries and conditions for selection, as discussed below.

Interaction Surface Presented by the $\mathbf{Z}_{\mathbf{S P A}-1}$ Affibody. Helices 1 and 2 in $Z_{\text {SPA-1 }}$ bind to helices 1 and 2 in $Z$ (Fig. 1). The overall topology can be described as a pseudosymmetric heterodimer of

(b) $\mathrm{CD}$ at $20^{\circ} \mathrm{C}$ of $\mathrm{Z}$ and $\mathrm{Z}_{\mathrm{SPA}-1}$ at $25 \mu \mathrm{M}$ concentrations, and of $\mathrm{Z}_{\text {SPA-1 }}$ at higher concentrations (as indicated) in $20 \mathrm{mM}$ potassium phosphate (pH 5.6). (c) Fluorescence emission spectra of $50 \mu \mathrm{M}$ ANS in buffer and in the presence of equimolar amounts of $Z, Z_{\text {SPA-1 }}$, and the $Z: Z_{\text {SPA-1 }}$ complex. All solutions contained $20 \mathrm{mM}$ potassium phosphate ( $\mathrm{pH}$ 5.6), and ANS was excited at $396 \mathrm{~nm}$. (d) Thermal melting of $\mathrm{Z}$ and $\mathrm{Z}_{\mathrm{SPA}-1}$ at $25 \mu \mathrm{M}$ monitored by $\mathrm{CD}$ at $208 \mathrm{~nm}$. 
a

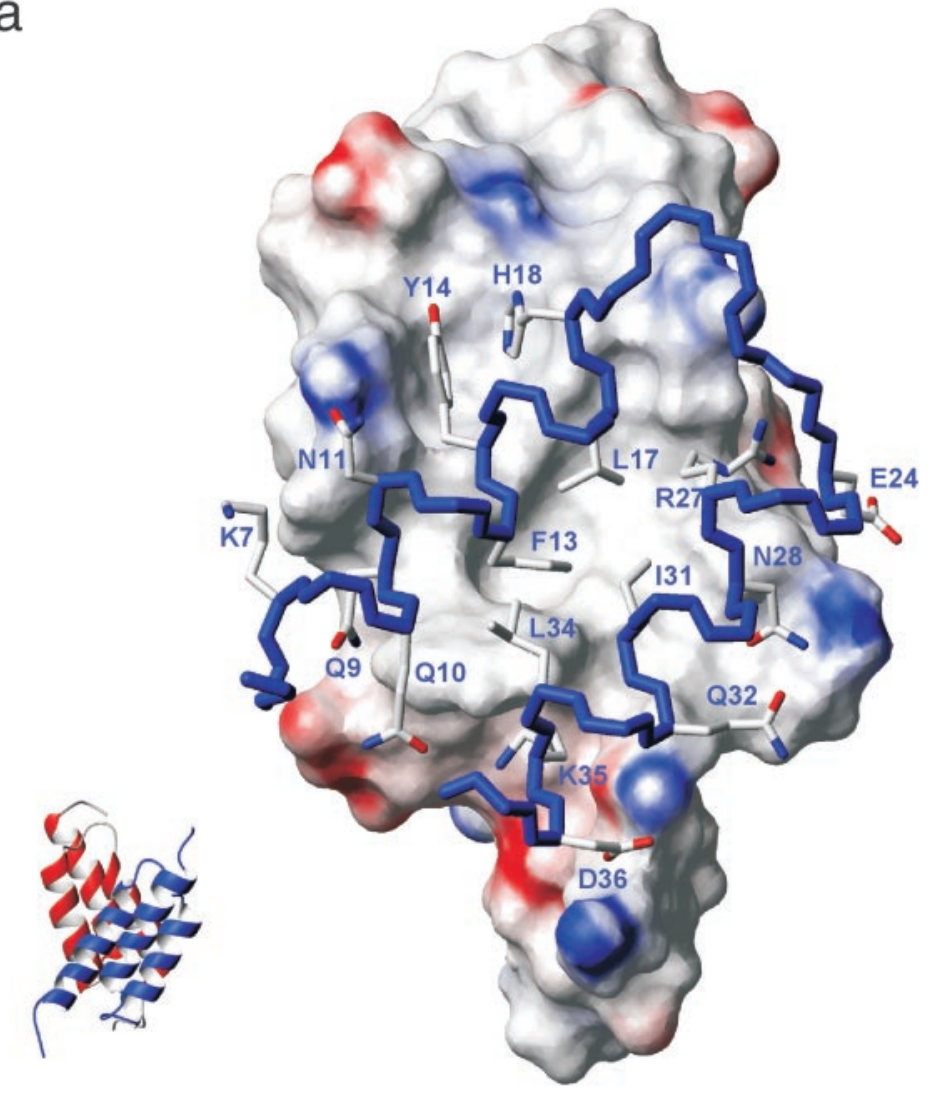

b

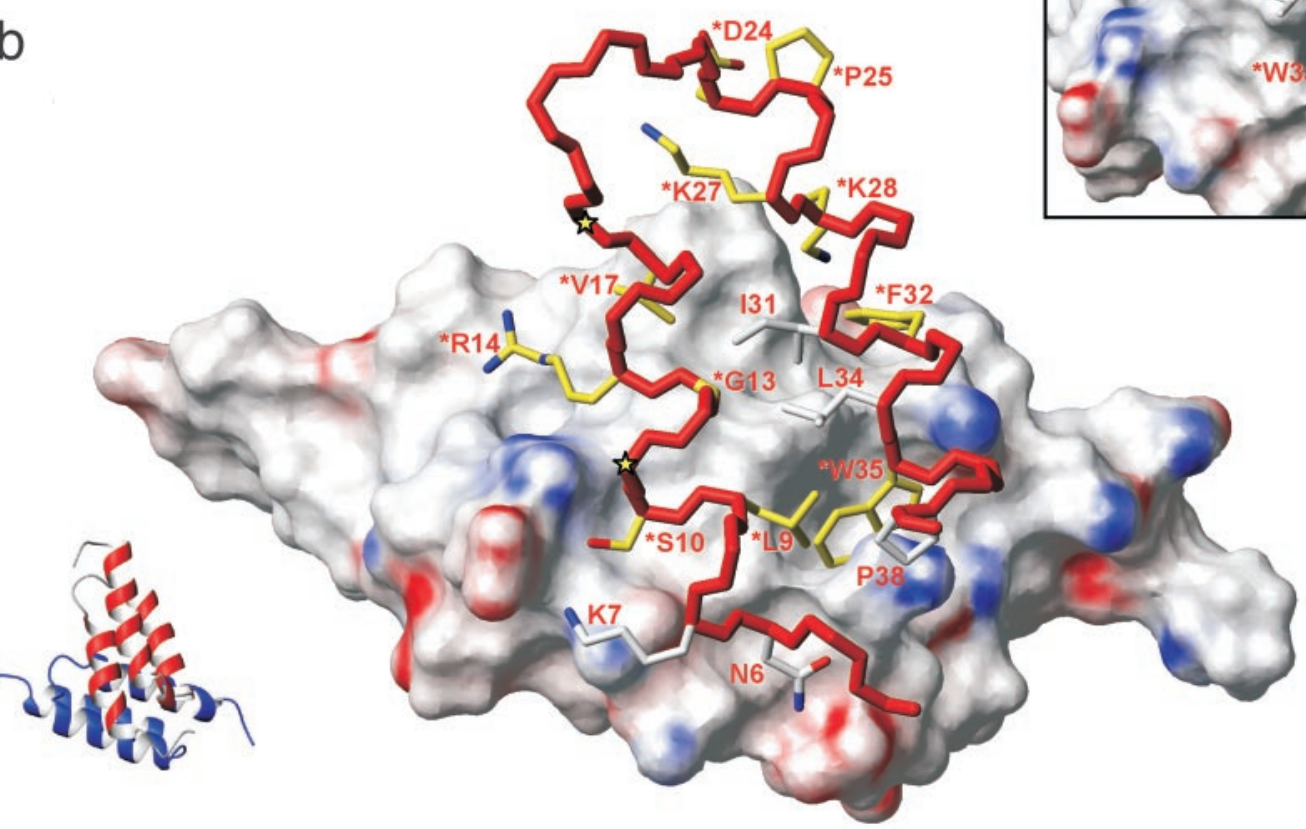

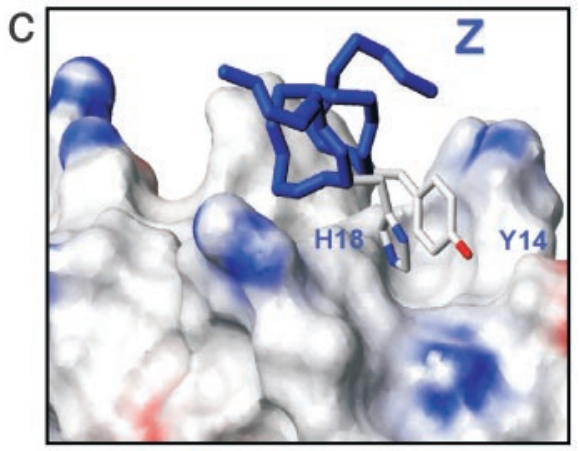

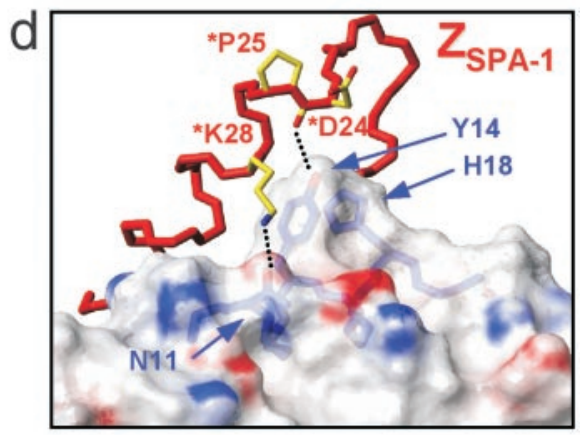

e

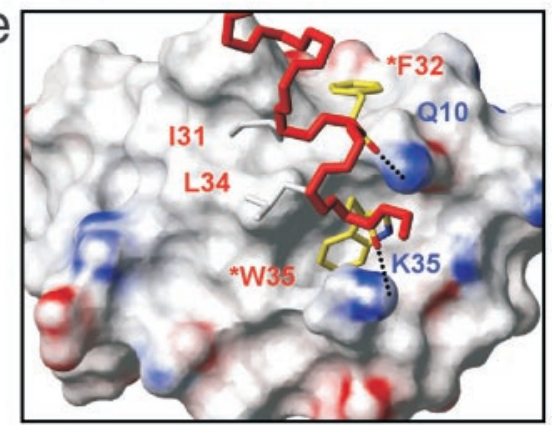

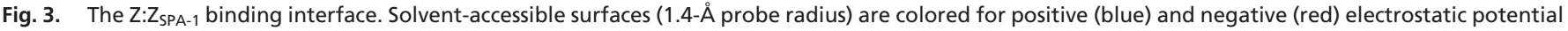

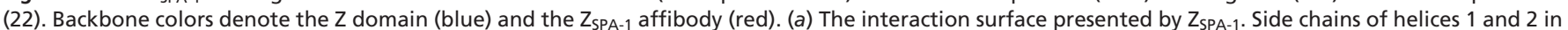

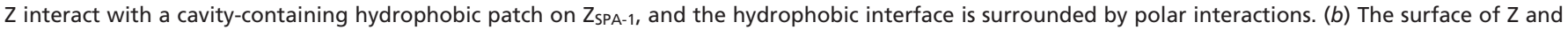

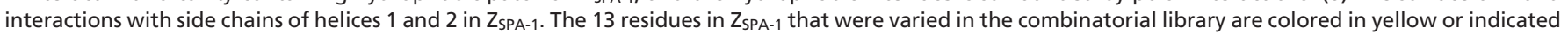

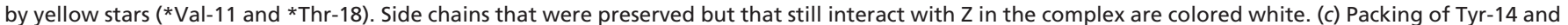

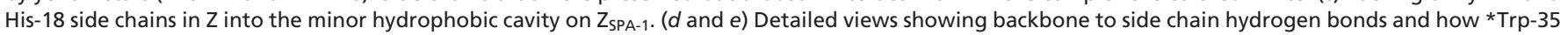
in $Z_{\text {SPA-1 }}$ is buried in a deep cavity on the $Z$ surface. Additional hydrogen bonds are described in the text.

two three-helix bundles. The interaction interface comprises $800-900 \AA^{2}$ of each subunit and is predominantly nonpolar $(64 \%)$ with polar interactions along solvent-exposed edges. The surfaces of helices 1 and 2 of $\mathrm{Z}_{\mathrm{SPA}-1}$ form a hydrophobic patch with two grooves (Fig. 3a). The larger hydrophobic groove is formed by *Leu-9, *Gly-13, *Val-17, Ile-31, *Phe-32, Leu-34, 
*Trp-35, and the aliphatic part of *Arg-14. These residues interact with Phe-13, Leu-17, Arg-27 (aliphatic part), Ile-31, and Leu-34 in the Z domain. The side chains of *Val-17, *Lys-27, *Lys-28, *Phe-32, and Ile-31 form a smaller hydrophobic groove with positively charged walls. The aromatic His-18 and Tyr-14 side chains in the $\mathrm{Z}$ domain are tightly packed against each other and fit precisely into the smaller groove, which is also flanked by the backbone of Asp-24 (in $\mathrm{Z}_{\mathrm{SPA}-1}$ ) to which Tyr-14 (in Z) donates a hydrogen bond (Fig. $3 c$ and $d$ ). The bulky side chain of *Trp-35 in $\mathrm{Z}_{\mathrm{SPA}-1}$ constitutes a large fraction of the interaction interface and is surrounded by six residues from helices 1 and 2 in the $\mathrm{Z}$ domain.

The edges of the interface contain multiple polar interactions and several hydrogen bonds. In addition to the Tyr-14 hydrogen bond, there are hydrogen bonds between the side chain of Gln-10 (in $\mathrm{Z}$ ) and the backbone carbonyl of *Phe-32 (in $\mathrm{Z}_{\mathrm{SPA}-1}$ ), between the backbone carbonyl of Leu-17 and the side chain of *Arg-14, and between the side chain of Lys-35 and backbone of *Trp-35. Glu-24 (in Z) and Lys-7 (in $\mathrm{Z}_{\mathrm{SPA}-1}$ ) appear to form a salt bridge. Some of these and other polar interactions are illustrated in Fig. $3 d$ and $e$.

Structural Basis for Selection of $\mathbf{Z}_{\mathrm{SPA}-1}$ as a Strong Binder to Protein $\mathbf{A}$. The interacting surface of the $\mathrm{Z}$ domain is characterized by two protrusions made by the Tyr-14/His-18 and Phe-13/Leu-17/Ile-31 side chains, respectively, which surround a hydrophobic groove, a deep cavity binding *Trp-35, and a shallow and mainly polar groove on the surface of helix 2 . $\mathrm{Z}_{\mathrm{SPA}-1}$ binds to this surface by direct contacts involving the side chains and/or backbone of 15 residues, of which 10 were allowed to vary in the combinatorial library (Fig. 3b). The basis for selection becomes clear on examination of these interactions. The replacement of Phe-13 (in Z) with the much smaller *Gly-13 contributes to the formation of the large hydrophobic cavity on $\mathrm{Z}_{\mathrm{SPA}-1}$ and tight packing interactions with Phe-13 and Leu-17 (in Z). The replacement of Lys-35 with *Trp-35 provides the possibility of binding into the deep hydrophobic cavity on $\mathrm{Z}$. This packing requires hydrophobic interactions also at the position of residue 32 , where a glutamine (in Z) is replaced by *Phe-32. Other examples include both polar and nonpolar interactions resulting from Tyr-14 to *Arg-14 and Asn-28 to *Lys-28 replacements. Several backbone carbonyls in $\mathrm{Z}_{\mathrm{SPA}-1}$ are also involved in binding (Fig. $3 d$ and $e$ ).

The replacement of Glu-25 (in Z) by *Pro-25 appears to play an indirect role, because the proline fixes the conformation of the preceding *Asp-24 backbone carbonyl in its hydrogen bond to the Tyr-14 hydroxyl (Fig. $3 d$ ). The replacements for which we cannot identify direct or indirect roles in recognition are Asn-11 to ${ }^{*}$ Val-11, His-18 to *Thr-18, and Glu-24 to *Asp-24. The two latter of these might be considered neutral due to similarities in size and polarity. Hence, the only potentially disadvantageous replacement in $\mathrm{Z}_{\mathrm{SPA}-1}$ appears to be $* \mathrm{Val}-11$, which contributes to the exposure of nonpolar surface on $Z_{\text {SPA-1 }}$ but does not appear to interact with the $\mathrm{Z}$ domain.

Interactions by side chains that are not allowed to vary in the affibody library can be understood in context of the contacts made by those that have been replaced. For instance, nonpolar interactions provided by *Leu-9, *Phe-32, and *Trp-35 bring neighboring Ile-31 and Leu-34 and also Pro-38 in $\mathrm{Z}_{\text {SPA-1 }}$ close to the $\mathrm{Z}$ surface, where they form part of the interface. The orientation of the interacting helices also allows for contacts by Asn-6 and Lys-7 (in $\mathrm{Z}_{\mathrm{SPA}-1}$ ), which presumably contribute to binding stability and specificity.

Induced Fit of the $\mathbf{Z}$ Domain Surface in Response to Binding of $\mathbf{Z}_{\text {SPA-1 }}$ or Fc. The binding surface of the uncomplexed $\mathrm{Z}$ domain (28) is rather flat, but it adopts a course and irregular morphology on binding to the $Z_{\text {SPA-1 }}$ affibody. In fact, the accessible surface on $\mathrm{Z}$ is almost $400 \AA^{2}$ larger in the bound than in the free state. This

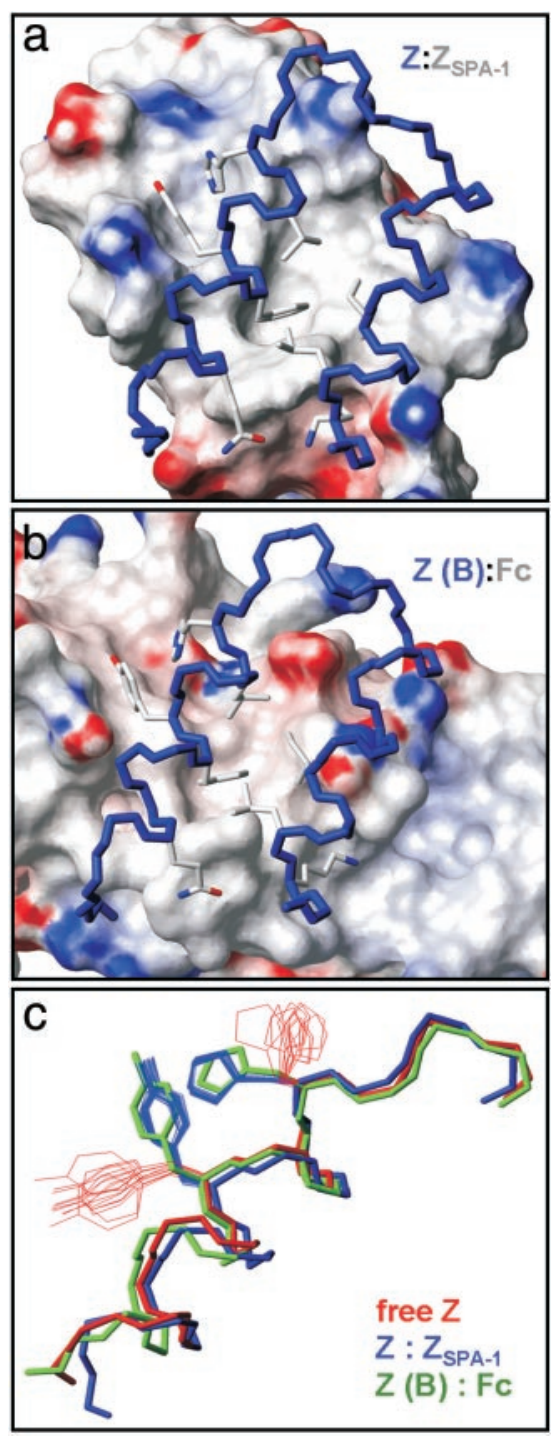

Fig. 4. Comparison of two SPA-binding surfaces and their interactions with the $Z$ domain. (a) Complex with the $Z_{\text {SPA-1 }}$ affibody. (b) Complex with Ig Fc (2). (c) The conformation of Tyr-14 and His-18 side chains in the free state (28) and in the two complexes illustrates that similar conformational changes (induced fit) occur.

additional surface constitutes almost half of the total interaction surface in the complex. Hence, it is valid to describe the formation of the $\mathrm{Z}: \mathrm{Z}_{\mathrm{SPA}-1}$ complex as a combination of coupled folding of the $Z_{\mathrm{SPA}-1}$ affibody and induced fit of side chains on the surface on the $\mathrm{Z}$ domain.

A comparison of the $\mathrm{Z}: \mathrm{Z}_{\mathrm{SPA}-1}$ complex with the complex between the B domain of SPA and an Ig Fc domain (2) reveals several similarities (Fig. 4). Both complexes contain hydrophobic intermolecular cores, which are flanked by polar interactions. Furthermore, there is a resemblance in the packing of $\mathrm{Z}$ (or B) domain side chains in the two complexes, for instance in the two protrusions on the $\mathrm{Z}$ surface and the deep hydrophobic cavity that binds $*$ Trp-35 from $Z_{\text {SPA-1 }}$, but which is filled by an isoleucine side chain in the complex with Fc. In fact, the conformational changes that Tyr-14 and His-18 in Z undergo on binding either $\mathrm{Z}_{\mathrm{SPA}-1}$ or $\mathrm{Fc}$ are almost identical (Fig. $4 c$ ). The explanation to $\mathrm{Z}_{\mathrm{SPA}-1}$ binding to the Fc-binding surface of $\mathrm{Z}$ is probably that the selection of $\mathrm{Z}_{\mathrm{SPA}-1}$ involved competitive phage elution by using human polyclonal $\operatorname{IgG}(8)$. However, it is 
intriguing that $\mathrm{Z}_{\mathrm{SPA}-1}$ mimics the IgG-binding surface, and that both proteins induce similar rearrangements of side chains on the $\mathrm{Z}$ surface.

Comparison to X-Ray Structure. A crystallographic structure of the $\mathrm{Z}: \mathrm{Z}_{\text {SPA-1 }}$ complex has been determined independently (29). The two structures are very similar in backbone conformation (rms deviation of $1.18 \AA$ for residues $5-56$ ) and in the packing of nonpolar side chains in the cores of the two proteins and at the protein-protein interface. There are a few differences in the polar interactions at the interface. For instance, the hydrogen bond between Tyr-14 (in Z) and backbone carbonyl of *Asp-24 is not observed in the $\mathrm{x}$-ray structure, in which the Tyr-14 hydroxyl in a slightly different conformation instead forms a water-mediated hydrogen bond to the *Asp-24 side chain. Two other differences include alternative side chain rotamers of Q32 and K35 (in Z) at or near the interaction interface. The packing of K35 against ${ }^{*} \mathrm{~W} 35$ in the NMR structure is supported by intermolecular NOEs, whereas the Q32 side chain orientation is consistent with NOEs to neighboring residues in the $\mathrm{Z}$ domain. The observed differences in polar interactions are not surprising considering the different dielectric environments provided by the high-salt solution in which the crystal was formed and the low-salt solution in which the NMR structure was determined.

Implications for Design and Selection of Binding Proteins. Molten globules sometimes occur in protein engineering (30-32). In the present case, it appears that the replacement of side chains on the surfaces of helices 1 and 2 in the $Z_{\text {SPA-1 }}$ affibody causes

1. Nilsson, B., Moks, T., Jansson, B., Abrahmsén, L., Elmblad, A., Holmgren, E., Henrichson, C., Jones, T. A. \& Uhlén, M. (1987) Protein Eng. 1, 107-113.

2. Deisenhofer, J. (1981) Biochemistry 20, 2361-2370.

3. Nord, K., Gunneriusson, E., Ringdahl, J., Stål, S., Uhlén, M. \& Nygren, P.-Å. (1997) Nat. Biotechnol. 15, 772-777.

4. Nord, K., Gunneriusson, E., Uhlén, M. \& Nygren, P.-Å. (2000) J. Biotechnol. 80, $45-54$.

5. Hansson, M., Ringdahl, J., Robert, A., Power, U., Goetsch, L., Nguyen, T., Uhlén, M., Ståhl, S. \& Nygren, P.-Å. (1999) Immunotechnology 4, 237-252.

6. Nord, K., Nord, O., Uhlén, M., Kelley, B., Ljungqvist, C. \& Nygren, P.-Å. (2001) Eur. J. Biochem. 268, 4269-4277.

7. Rönnmark, J., Grönlund, H., Uhlén, M. \& Nygren, P.-Å. (2002) Eur. J. Biochem. 269, 2647-2655.

8. Eklund, M., Axelsson, L., Uhlén, M. \& Nygren, P.-Å. (2002) Proteins Struct. Funct. Genet. 48, 454-462.

9. Lendel, C., Wahlberg, E., Berglund, H., Eklund, M., Nygren, P.-Å. \& Härd, T. (2002) J. Biomol. NMR 24, 271-272.

10. Wagner, G., Hyberts, S. G. \& Havel, T. F. (1992) Annu. Rev. Biophys. Biomol. Struct. 21, 167-198.

11. Vuister, G. W., Kim, S.-J., Wu, C. \& Bax, A. (1994) J. Am. Chem. Soc. 116, 9206-9210.

12. Cavanagh, J., Fairbrother, W. J., Palmer, A. G., III, \& Skelton, N. J. (1996) Protein NMR Spectroscopy: Principles and Practice (Academic, New York).

13. Helgstrand, M., Kraulis, P., Allard, P. \& Härd, T. (2000) J. Biomol. NMR 18, 329-336.

14. Wüthrich, K. (1986) NMR of Proteins and Nucleic Acids (Wiley, New York).

15. Fletcher, C. M., Jones, D. N. M., Diamond, R. \& Neuhaus, D. (1996) J. Biomol. NMR 8, 292-310. destabilization of the three-helix bundle. This is most likely unfavorable for binding affinity, because some of the free energy gained on formation of a complex is needed to fold the affibody. To improve affibody stability and thereby binding affinity, it might be advantageous to restrict the possibilities that polar side chains at the edges of the binding surface are replaced by nonpolar side chains. For instance, ${ }^{*}$ Val-11 in $\mathrm{Z}_{\mathrm{SPA}-1}$, which replaces Asn-11 in Z, does not appear to contribute to binding. (That*Val-11 appears in the selected protein might be due to the limited size of the phage display library, which includes only a small subset of all $20^{13}$ possible variants.) The completely folded state of free $Z_{\text {SPA-1 }}$ cannot be observed, but it is likely to be very similar to that of the $\mathrm{Z}$ domain. The replacement at position 11 is therefore expected to have a destabilizing effect, because it results in the substitution of polar surface with nonpolar surface. It is of course difficult to deduce a priori which side chain replacements need to be restricted. However, this problem might possibly be overcome if additional affibody-target structures reveal some generalities or if sequence comparisons of a larger number of affibodies reveal common recurrences. A second and more easily implemented method to improve affibody stability is to carry out the selection at conditions that favor stably folded proteins without impairing phage viability, for instance by panning at higher temperature or in the presence of chemical denaturants.

We thank Dr. Johan Weigelt at Biovitrum for assistance. P.-A..N. is cofounder and consultant for Affibody AB (Bromma, Sweden). This work was supported by the Knut and Alice Wallenberg Foundation and the Swedish Foundation for Strategic Research.

16. Cornilescu, G., Delaglio, F. \& Bax, A. (1999) J. Biomol. NMR 13, 289-302.

17. Ludvigsen, S. \& Poulsen, F. M. (1992) J. Biomol. NMR 2, 227-233.

18. Jeffrey, G. A. \& Saenger, W. (1991) Hydrogen Bonding in Biological Structures (Springer, Berlin).

19. Brünger, A. T., Clore, G. M., Gronenborn, A. M. \& Karplus, M. (1986) Proc. Natl. Acad. Sci. USA 83, 3801-3805.

20. Kuszewski, J., Gronenborn, A. M. \& Clore, G. M. (1999) J. Am. Chem. Soc. 121, 2337-2338.

21. Kuszewski, J., Gronenborn, A. M. \& Clore, G. M. (1997) J. Magn. Reson. 125, 171-177.

22. Koradi, R., Billeter, M. \& Wüthrich, K. (1996) J. Mol. Graphics 14, 51-55.

23. Laskowski, R. A., Rullmann, J. A. C., MacArthur, M. W., Kaptein, R. \& Thornton, J. M. (1996) J. Biomol. NMR 8, 477-486.

24. Rodriguez, R., Chinea, G., Lopez, N., Pons, T. \& Vriend, G. (1998) CABIOS 14, 523-528.

25. Semisotnov, G., Rodionova, N., Razgulyaev, O., Uversky, V., Gripas, A. \& Gilmanshin, R. (1991) Biopolymers 31, 119-128.

26. Dobson, C. (1994) Curr. Biol. 4, 636-640.

27. Ptitsyn, O. (1995) Trends Biochem. Sci. 20, 376-379.

28. Tashiro, M., Tejero, R., Zimmerman, D. E., Celda, B., Nilsson, B. \& Montelione, G. T. (1997) J. Mol. Biol. 272, 573-590.

29. Högbom, M., Eklund, M., Nygren, P.-A. \& Nordlund, P. (2003) Proc. Natl. Acad. Sci. USA 100, 3191-3196.

30. Aphasizheva, I., Dolgikh, D., Abdullaev, Z., Uversky, V., Kirpichnikov, M. \& Ptitsyn, O. (1998) FEBS Lett. 425, 101-104.

31. Lecomte, J. \& Matthews, C. (1993) Protein Eng. 6, 1-10.

32. Tahmassebi, D. C. \& Sasaki, T. (1998) J. Org. Chem. 63, 728-731. 